DOI: /((please add manuscript number $))$

Article type: VSI EMRS Laser materials

\title{
Localized infrared radiation-induced hyperthermia sensitized by laser- ablated silicon nanoparticles for phototherapy applications
}

V. A. Oleshchenko , A. Yu. Kharin ${ }^{\mathrm{b}}$, A. F. Alykova ${ }^{\mathrm{b}}$, O. V. Karpukhina ${ }^{\mathrm{c}, \mathrm{d}}$, N. V. Karpov ${ }^{\mathrm{b}}$, A. A. Popov ${ }^{\text {b }, ~ V . ~ V . ~ B e z o t o s n y i ~}{ }^{\mathrm{a}}$, S. M. Klimentov ${ }^{\mathrm{b}}$, I. N. Zavestovskaya ${ }^{\mathrm{a}, \mathrm{b}}$,

A. V. Kabashin ${ }^{b, e *}$, V. Yu. Timoshenko ${ }^{a, b, f *}$

${ }^{a}$ Lebedev Physical Institute of the Russian Acad. Sci., Leninskiy Pr. 53, 119991

Moscow, Russia

'National Research Nucler University "MEPhI", Institute of Engineering Physics for

Biomedicine (PhysBio), Kashirskoe sh. 31, 115409 Moscow, Russia

${ }^{\mathrm{c}}$ Lomonosov Moscow State University, Faculty of Biology, Leninskie Gory 1, 119991, Moscow, Russia

${ }^{d}$ Semenov Institute of Chemical Physics of the Russian Acad. Sci., 119991 Moscow, Russia

eAix-Marseille University, CNRS, LP3, 163 Avenue de Luminy, 13288 Marseille, France

${ }^{f}$ Lomonosov Moscow State University, Faculty of Physics, Leninskie Gory 1, 119991

Moscow, Russia

\section{Highlights:}

- Laser-ablated silicon nanoparticles act as sensitizers for spatially and temporally localized photohyperthermia under irradiation in the tissue transparency window.

- Irradiation with millisecond pulses of laser diode promotes hyperthermia effect due to non-stationary temperature distribution nearby silicon nanoparticles.

- In vitro experiments confirm the hyperthermia sensitized by silicon nanoparticles.

*To whom the correspondence should be addressed: kabashin@1p3.univ-mrs.fr, vtimoshe@gmail.com 


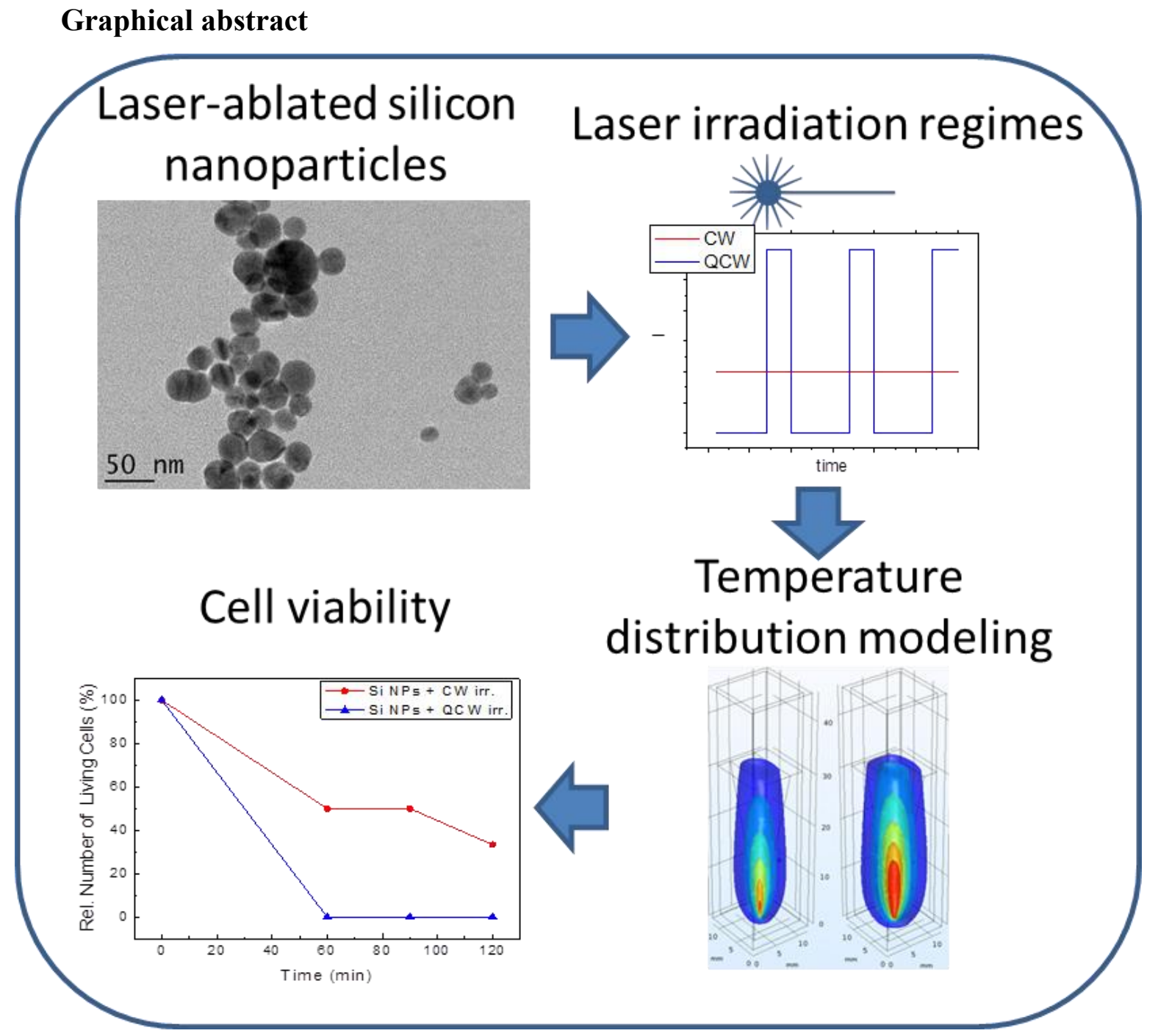




\begin{abstract}
Silicon (Si) nanoparticles (NPs) synthesized by methods of laser ablation in water are explored as sensitizers of photothermal therapy under a laser excitation in the window of relative tissue transparency. Based on theoretical calculations and experimental data, it is shown that the NPs can be heated up to temperatures above $42-50^{\circ} \mathrm{C}$ by laser diode irradiation at 808 $\mathrm{nm}$ in continuous wave $(\mathrm{CW})$ and quasi-continuous wave (QCW) regimes. Profiting from the laser-induced heating, a high efficiency Si-NPs as sensitizers of the hyperthermia of cells in Paramecium Caudatum model is demonstrated. The QCW regime is found to be more efficient, leading to complete cell destruction even under relatively mild laser irradiation conditions. The obtained data evidence a great potential in using laser-ablated Si-NPs as sensitizers of photohyperthermia in antibacterial or cancer therapy applications.
\end{abstract}

\title{
Keywords
}

Pulsed laser ablation in liquids, Nanoparticles; Silicon; Laser diode; Photoexcitation; Hyperthermia; Paramecium Caudatum 


\section{Introduction}

Silicon ( $\mathrm{Si}$ ) nanoparticles (NPs) exhibit a series of properties, which make them extremely promising for biomedical applications $[1,2,3]$. Si nanocrystals with sizes below $10 \mathrm{~nm}$ possesses photoluminescence in the window of relative tissue transparency $(650-850 \mathrm{~nm})$, which can be used for their detection in biological cells [4,5] and tissues [6]. On the other hand, Si-NPs can offer sensitizing properties to initiate therapies under external stimuli, including photodynamic therapy (PDT) [7], ultrasound [8], radiofrequency (RF) radiation- [9] and infrared (IR) radiation-induced [10] hyperthermia. However, biomedical prospects of nanosilicon structures are complicated by the difficulty of their synthesizing in pure, uncontaminated and waterdispersible form. Indeed, conventional wet chemical routes for the fabrication of Si-NPs cannot normally avoid the use of toxic substances and by-products, such as non-saturated organic compounds [11] or solvents [12], while Si-NPs synthesized by "dry" routes are typically not water dispersible $[13,14]$ and require similar wet chemical steps to enable this option. Porous NPs prepared by mechanical milling of electrochemically fabricated porous Si seem to be well suitable for biomedical applications [1,15], but such NPs can be contaminated by hydrofluoric acid derivatives [16] and present a widely size- and shape-dispersed population [15] complicating their transport in vivo.

Si-NPs prepared by methods of laser ablation in gaseous [17,18] or aqueous [19-21] ambient present a novel object, which promises the solution of the above-stated toxicity and other problems. When produced in ultrapure, well-controlled ambient (residual helium gas, deionized water), laser-synthesized nanostructures are free of any toxic impurities and water-dissolvable with the dissolution rate controllable by conditions of laser-ablative synthesis [22]. As follows from results of our recent studies, a systemic administration of Si-NPs in a small animal model does not provoke any toxicity effects, while the NPs rapidly biodegrade and excrete from the body within several days [23]. More importantly, laser-synthesized Si-NPs can initiate a series of bioimaging and therapy functionalities based on their unique physico-chemical properties $[24,25]$. As an example, small (2-4 nm) Si nanocrystals can enable imaging functionality based on the photoluminescence (PL) via quantum confined excitonic states [6], while larger NPs (10$100 \mathrm{~nm}$ ) can offer dual non-linear bioimaging modality based on the combination of Second Harmonic Generation (SHG) and Two-Photon Excited Luminescence (TPEL) channels [26]. On the other hand, laser-synthesized Si NPs can be efficient sensitizers of therapeutic modalities such as PDT [7] and RF radiation-induced hyperthermia [9], as well as serve as carriers of therapeutic radionuclides [27]. Such a combination of different modalities in one Si-based nanoformulation looks as an effective strategy to develop novel theranostic (therapy + 
diagnostics) agents for biomedical applications. In this sense, additional diagnostic and therapeutic functionalities based on laser-synthesized NPs can be considered as new important assets.

In this paper, we show that laser-synthesized Si-NPs can serve as efficient sensitizers of infrared radiation-induced hyperthermia similarly to how it was earlier reported for electrochemically produced porous silicon-based NPs $[10,28]$. We suppose that such a modality can be used in parallel with other approaches to improve the efficiency of antibacterial and cancer treatments.

\section{Materials and methods}

Crystalline silicon (c-Si) wafers having resistivity of 10-20 $\mathrm{Ohm} \cdot \mathrm{cm}$ and crystallographic surface orientation (100) were used as targets for laser ablation experiments. The wafers were treated with an aqueous solution of HF (48\%) for 1-2 s to remove a natural silicon oxide from the surface. In laser ablation experiments, we used a linearly polarized beam from a diode pump Teta 10 system (Avesta, Russia), operating at wavelength of $1030 \mathrm{~nm}$, pulse length $270 \mathrm{fs}$, pulse energy $100 \mu \mathrm{J}$, repetition rate $10 \mathrm{kHz}$. A laser beam ( $3 \mathrm{~mm}$ in diameter) was focused onto target immersed in $10 \mathrm{ml}$ of deionized water at normal incidence. The experiment was carried out for $1 \mathrm{~h}$ at room temperature. Aqueous suspensions of laser-synthesized Si-NPs having initial concentration of $0.1 \mathrm{mg} / \mathrm{mL}$ were centrifuged (12000 g, $20 \mathrm{~min})$ until the concentration reached $4 \mathrm{mg} / \mathrm{mL}$.

Diluted suspensions of Si-NPs were investigated by transmission electron microscopy (TEM) using a LEO912 AB OMEGA system. Dissolution kinetics for diluted Si-NPs solutions in media having different $\mathrm{pH}$ were investigated by optical extinction spectroscopy using Cary $60 \mathrm{UV}-\mathrm{Vis}$ spectrometer. Size and charge characteristics of Si-NPs in aqueous suspension were studied by dynamic light scattering (DLS) using a Malvern Zetasizer ZS analyzer.

To carry out photothermal and biological tests, we used a home-made laser diode providing $808 \mathrm{~nm}$ radiation at the average power of $3 \mathrm{~W}$ in continuous wave $(\mathrm{CW})$ or quasi-continuous wave (QCW) regimes [29]. The QCW mode provided periodic $0.3 \mathrm{~s}$ pulses at power of $10 \mathrm{~W}$ followed by $0.7 \mathrm{~s}$ offset mode. The laser irradiation was applied from the bottom side of a transparent plastic cuvette having a cross-sectional size of $10 \times 10 \mathrm{~mm}^{2}$ and the length of $40 \mathrm{~mm}$ (inset in Figure 1b). Laser beam cross-section area at the cuvette bottom was about $0.02 \mathrm{~mm}^{2}$. A 1-mm in diameter temperature sensor was located at $15 \mathrm{~mm}$ from the bottom of the cell, in one of its corners, to avoid direct laser irradiation. 
To analyze toxicity of Si NPs and assess effects of continuous and pulsed laser illumination, we used suspensions of living cells of Paramecium Caudatum. Despite the simplicity of organization, this type of cells combines features of both a single cell and the whole organism [30]. These cells are interesting from analytical point of view as they can be considered as simple receptor systems that have the ability to respond to thermal effects of a whole set of biological, physiological and biochemical stimuli [31,32]. As an example, the movement velocity of Paramecium Caudatum cells drastically increases when temperature is increased from 25 to $30^{\circ} \mathrm{C}$, while a further increase of temperature above $40^{\circ} \mathrm{C}$ typically leads to the cell death $[32,33]$. The latter temperate is close to the well-known hyperthermia threshold for cancer cells (see for example review in Ref. [34]). Prior laser irradiation, NPs aqueous suspension (1 $\mathrm{mL}$ with concentration $1.2 \mathrm{mg} / \mathrm{mL}$ ) was mixed with a cell suspension ( $2 \mathrm{~mL}$ ) in a plastic cuvette, and the mixture was incubated for $30 \mathrm{~min}$. The cell death was visually estimated by the absence of cell movement, often associated with the deformation and lysis of the cell wall. As a control test, we monitored the number of cells in $10 \mathrm{ml}$ of the medium containing the intact culture of infusoria. The evolution of temperature of aqueous suspensions under laser irradiation was monitored by using a digital medical thermometer TMTSP-1 (MIREA, Russia) having a temperature sensor tip from the Exacon Scientific (Denmark). The range of thermometer measurements was between +5 and $+50^{\circ} \mathrm{C}$, measurement accuracy and sampling rate were $0.01^{\circ} \mathrm{C}$ and $15 \mathrm{~Hz}$, respectively. In order to avoid direct heating of the temperature sensor under laser irradiation, the sensor was located at the periphery of the cuvette outside the irradiation region.

\section{Calculations}

In order to access temperature in the center of cuvette filled with NPs solutions, a threedimensional finite-element model was developed with using COMSOL Multiphysics software. Temperature distribution was modeled via a numerical solution of the thermal conductivity equation:

$$
\frac{\partial T(x, y, z, t)}{\partial t}=D \cdot \Delta T(x, y, z, t)+H(x, y, z, t),
$$

where $\mathrm{D}$ is the thermal diffusion coefficient $\left(D=1.43 \cdot 10^{-7} \mathrm{~m}^{2} / \mathrm{s}\right.$ for water at $\left.25^{\circ} \mathrm{C}\right)$ and $H(x, y, z, t)$ is the heat generation source function. The boundary conditions consider constant temperature of cuvette walls (corresponding to ambient temperature), and presence of air above the liquid in the cuvette. Geometry of the cuvette and laser beam divergence were set in the form of an inverted truncated pyramid with height, base, and area of the initial cross-section 
corresponding to experimental conditions. While the laser beam propagates along the $\mathrm{z}$ coordinate, the heat source can be approximated as follows:

$$
H(x, y, z, t) \approx P(t) K(x, y, z) \frac{k \cdot \exp (-k \cdot z)}{\left(x_{0}+z \cdot \operatorname{tg} \alpha\right)\left(y_{0}+z \cdot \operatorname{tg} \alpha\right)},
$$

where $P(t)$ is the excitation power at the cuvette entrance, $K(x, y, z)$ is the dimensionless factor, which describes the heat source geometry, in which $K=1$ inside and $K=0$ outside of the excitation region. $\mathrm{x}_{0}=0.1 \mathrm{~mm}$ and $\mathrm{y}_{0}=0.2 \mathrm{~mm}$ are dimensions of the cross section of the laser beam at the cuvette entrance, and $\alpha \approx 18^{\circ}$ is the laser beam divergency angle, which was determined experimentally. The numerator shows the absorbed light intensity in accordance to Beer-Lambert law, where $k$ is a linear absorbance coefficient for current concentration of nanoparticles at wavelength $808 \mathrm{~nm}$. The denominator shows the area of rectangular beam cross-section at height $\mathrm{z}$.

\section{Results and discussion}

A typical TEM image of dried Si-NPs and a corresponding electron diffraction pattern are shown in Figure 1a. One can see that the formed NPs had an ideal spherical shape, while their mean size was about 20-30 nm. As follows from electron diffraction data (inset of Fig. 1a), SiNPs had a polycrystalline structure. As shown from DLS spectrum from as-prepared suspension of Si-NPs (Figure 1b), mean hydrodynamical size (diameter) and zeta-potential of the NPs were about $100 \mathrm{~nm}$ and $-15 \mathrm{mV}$, respectively. A larger value of the hydrodynamic size compared to the TEM data can be explained by the appearance of an ionic shell around Si-NPs in aqueous media. The negative zeta-potential of the prepared Si-NPs was obviously related to the presence of hydroxyl groups on the Si-NPs surface [22, 23]. Structural and dissolution properties of laserablated Si-NPs were studied in our earlier works [9, 19, 20, 22]. According to Fourier transform infrared spectroscopy (FTIR) [9] and X-ray photoelectron spectroscopy (XPS) [20, 22] data, the upper layer of laser-ablated Si-NPs is oxidized, with a certain amount of hydrogen still remaining on the NPs surface. Such a silicon oxide-related coating of Si-NPs is supposed to condition hydrophilic properties of their surface. It was also found from Raman spectroscopy and TEM data $[9,22]$ that laser-ablated Si-NPs could completely dissolve in physiological environment. In this case, the dissolution rate depended on whether laser ablation was performed in normal oxygen-saturated water (oxygen-rich conditions) or in water deoxygenated by pumping with noble gases ( $\mathrm{Ag}$ or $\mathrm{He}$ ) before and during the experiment (oxygen-free conditions) [22]. Here, more oxidized Si-NPs prepared in oxygen-rich conditions demonstrated much faster dissolution kinetics (4-7 days compared to 3-4 weeks), which was explained by the relative friability of the oxygen defect-rich nanoparticle core [22]. 
To access photohyperthermia applications of laser-ablated Si-NPs, it is important to check the stability of these nanostructures in projected biological conditions. Therefore, we first explored dissolution properties of Si-NPs in aqueous solutions having different acidity, i.e. $\mathrm{pH}$ level. Figure 2a shows extinction spectra of Si-NPs (as-prepared and aged in water buffer at pH7). As shown in the Figure, the extinction peak dropped 10-fold in intensity after its aging in $\mathrm{pH} 7$ buffer for 10 days, evidencing the dissolution process of Si-NPs. Based on extinction spectra data, we then measured kinetics of NPs dissolution in $\mathrm{pH} 5, \mathrm{pH} 7, \mathrm{pH} 8$ buffers (Figure $2 \mathrm{~b}$ ). As shown in the figure, the degradation rate depended on $\mathrm{pH}$, with higher rates for solutions having higher $\mathrm{pH}$, while characteristic degradation times for all samples were in the range of several days. It should be noted that the degradation time was much longer than projected laser treatment times (tens of minutes) and longer than typical times of NPs accumulation in vivo [23]. Such a degradation kinetics looks favorable for applications of laser-synthesized Si-NPs in bioimaging and therapies.

Figure 3 shows simulated kinetics of temperature growth during laser heating of Si-NP colloids under CW (a) and QCW (b) irradiation regimes. Here, the extinction coefficient $\mathrm{k}=$ $0.21 \mathrm{~cm}^{-1}$ for $808 \mathrm{~nm}$ wavelength under concentration of $0.4 \mathrm{~g} / \mathrm{L}$ was found experimentally, while the extinction coefficient of pure water was below $0.05 \mathrm{~cm}^{-1}$ under the same laser irradiation conditions. Red and blue curves in panels (a) and (b) show temperature at different distances from the cuvette bottom. Two modes, CW and QCW had the same average power of $3 \mathrm{~W}$, but the QCW mode had a higher instant power $(10 \mathrm{~W})$ as it comprised a series of rectangular pulses having the peak power of $10 \mathrm{~W}$, duration of $0.3 \mathrm{~s}$ and frequency of $1 \mathrm{~Hz}$. One can see that the irradiation in QCW mode led to temperature peaks that exceeded the temperature level in $\mathrm{CW}$ mode by $3-5^{\circ} \mathrm{C}$. One can also mention that temperature peaks became smoothened at peripheral parts of the cuvette due to a low thermal diffusivity of water (CW and QCW modes have the same effect). Color insets in panels (a) and (b) of Figure 3 show simulated images of isothermal surfaces inside the cuvette after the irradiation for $5 \mathrm{~min}$ in $\mathrm{CW}$ and QCW modes. Here, a red isothermal surface corresponded to heating by more than $30{ }^{\circ} \mathrm{C}$ relative to the initial temperature. It is visible that in QCW mode this temperature was recordable even at points located $10 \mathrm{~mm}$ from the bottom (b), while in $\mathrm{CW}$ mode the relevant temperature could be reached only for close points $(<3 \mathrm{~mm}$ ). In general, our calculations showed that QCW mode looked much more efficient for the implementation of local hyperthermia under the same values of the average power $(3 \mathrm{~W})$.

The simulated effect of local heating of aqueous NPs suspensions under their irradiation at $808 \mathrm{~nm}$ can be explained by particular properties of laser-synthesized Si-NPs. Since the 
measured extinction coefficient of about $0.5 \mathrm{~cm}^{-1}$ (for $1 \mathrm{~g} / \mathrm{L}$ concentration of NPs) can hardly be related to phonon-assisted electron transitions in indirect band gap silicon, we can attribute such enhanced optical absorption to local field effects at the nanoscale [26] or/and electron defect states in Si-NPs. The involvement of the first effect was confirmed by the appearance of non-monotonic features in the extinction spectra (Fig. 2a), evidencing Mie resonances in laserablated Si-NPs. Possible contribution of defect states follows from defect-rich structure of lasersynthesized Si-NPs as a result of their formation under strongly non-equilibrium aqueous conditions [22]. Indeed, such Si-NPs presented a polycrystalline structure comprising numerous intrinsic defects such as twins and grain boundaries (Fig. 1a).

Figure 4 shows time dependencies for temperature of aqueous suspensions of Paramecium Caudatum cells alone and the cells with incubated Si-NPs under CW (a) and QCW (b) laser irradiation. As shown in the figure, the temperature increase of the cell suspension incubated with Si-NPs was much more pronounced compared to the cells without NPs, while the mean temperature growth was similar for the $\mathrm{CW}$ and QCW irradiation regimes. This fact indicates that Si-NPs acted as efficient absorbers of the applied near-infrared laser radiation. Here, a direct measurement of the absorbed power revealed the extinction coefficient about $\mathrm{k}=0.21$ $\mathrm{cm}^{-1}$ for the suspension of $0.4 \mathrm{mg} / \mathrm{ml} \mathrm{Si-NPs}$. It is also important that maximal temperature achieved at the peak of laser pulse in the QCW regime significantly exceeded the mean temperature in this regime (inset of Fig. 4b). Note that the absolute value of the temperature growth during the action of laser pulse was underestimated because of a limited time resolution and peripheral location of the thermometer used.

Finally, we carried out a series of cell viability measurements in order to assess the efficiency of laser-induced treatment using Si-NPs as sensitizers of local photo-hyperthermia. Such tests revealed a significant difference in the cell survival rates after applying CW and QCW irradiation modes. Indeed, the application of QCW mode led to a complete destruction of Paramecium Caudatum cells with incubated Si-NPs after 60 min irradiation, while $50 \%$ of cells were still alive after similar application of the CW mode (Figure 5). Before laser irradiation, Paramecium Caudatum cells presented unicellular organisms, which freely moved in the liquid medium (Fig. 6a, this image was taken by a fast snapshot of moving object), while after laser irradiation in the QCW mode $\left(0.3 \mathrm{~s}\right.$ pulses, peak power density $\left.100 \mathrm{~W} / \mathrm{cm}^{2}\right)$ they were dead stationary objects (Fig. 6 b,c). Just after the first pulse, we observed a change in the nature of cell movement, while after the third pulse we could detect only slow motions over a circular trajectory. Then, after 6-7 pulses the movement nearly stopped. Comparing images in panels (a) and (b) of Figure 6, one can reveal changes in the shape of microorganisms and their internal 
structure. Before laser irradiation, the shape was elongated; the internal structure had a clear outline. After the irradiation, the elongation became shapeless, while the internal structure was broken (intracellular organelle contrast was still high due to NPs accumulation inside the cells). The cell destruction is illustrated in Fig. 6 b,c by snapshots taken in $60 \mathrm{sec}$ and 180 -sec intervals after the exposition. Within 3 minutes of observation after the exposition digestive vacuoles inside the cells increased in size, while the cell volume decreased probably due to a partial destruction of the membrane as the contrast of the cell membrane became significantly reduced. The thermolysis of the cell membrane and its interior was the most probable reason of cell death under hyperthermia conditions. In our tests, the threshold power density for cells death was below $100 \mathrm{~W} / \mathrm{cm}^{2}$ even for relatively low NPs concentrations $(<0.08 \mathrm{~g} / \mathrm{L})$. For comparison, we carried out cell irradiation tests in the absence of Si-NPs. Step-by-step increasing laser power density we managed to evaluate the "threshold" laser intensity, which can provide cell death in water without the NPs. This threshold intensity was about $150 \mathrm{~W} / \mathrm{cm}^{2}$, which was much higher than in the case of NPs. After the treatment by a single laser pulse at this intensity, microorganisms were alive exhibiting a very slow movement for 30-50 minutes, while the application of many pulses led to a gradual decrease of the movement ability and a subsequent cell death. Our analysis showed that in the absence of Si-NPs laser-induced treatment damages the most absorbing, contrasting cell organelles such as macronucleus, micronucleus, and digestive vacuoles. In general, the sensitizing effect of Si NPs was pronounced making possible the destruction of cells under much lower radiation intensities.

Thus, laser-synthesized Si-NPs can be efficient sensitizers of local overheating under nearinfrared laser excitation. As follows from our tests in Paramecium Caudatum cell model, the QCW mode appeared to be more efficient in the elimination of cells with the cell death reaching $100 \%$ under 60 min irradiation at the mean power of $3 \mathrm{~W}$. A better efficiency of QCW mode can be explained by a very high peak power at the maxima of laser pulses $(10 \mathrm{~W})$, which caused a major damage to the cells. We believe that the proposed photo-induced hyperthermia sensitized by laser-ablated Si-NPs can be used solely or in combination with other approaches profiting from the unique physical and chemical properties of Si-based nanostructures. On the one hand, because the mean size of laser-ablated Si is usually above the quantum confinement regime, they are hardly applicable in the conventional fluorescent bioimaging [1-4]. On the other hand, Si-NPs with sizes above $10 \mathrm{~nm}$ can be visualized by means of the nonlinear optical bioimaging in the TPEL and SHG modes [26]. Moreover, the NP-sensitized hyperthermia is well combined with earlier demonstrated alternative therapy methods, including the RF- 
induced hyperthermia [9] and PDT [7], which could be enabled in parallel profiting from the sensitizing properties of Si-NPs.

We believe that the use of laser-synthesized Si-NPs as sensitizers of photo hyperthermia can offer several advantages compared to porous silicon and other nanosilicon-based counterparts: (i) In contrast to porous silicon nanostructures, laser-synthesized Si-NPs have ideal spherical shape and are low size-dispersed, which promises a much improved transport in biological systems in vivo [27]; (ii) Laser-synthesized Si-NPs can have a defect-rich polycrystalline structure, which conditions a much faster dissolution and excretion of these nanoparticles from the body in vivo (4-7 days [22,23] compared to more than 6 weeks in the case of porous silicon [35]); (iii) Laser-synthesized Si-NPs are produced in ultrapure deionized water, which excludes any contamination of nanoparticles by toxic by-products of synthesis, as it takes place in many alternative methods $[11,12,16]$; (iv) Laser-synthesized Si-NPs can offer a series of imaging $[5,26]$ and therapy (Refs. $[9,19,27]$ ) modalities, which can be enabled in parallel with the considered photothermal modality.

\section{Conclusions}

We showed that laser-synthesized Si NPs can be efficient sensitizers of local hyperthermia under their illumination by laser radiation in the region of relative tissue transparency $(808 \mathrm{~nm})$. As follows from both the calculations and experimental results, the millisecond pulses QCW regime appeared to be advantageous compared to the $\mathrm{CW}$-based one to reach higher temperatures under a high spatial localization of the heating release. The efficiency of hyperthermia-based phototherapy therapy was illustrated for the model of Paramecium Caudatum cells. Here, our experiments confirmed that the QCW regime was indeed more efficient to initiate the hyperthermia leading to $100 \%$ cell death even for relatively low NPs concentrations.

The presented simplified approach can be the basic step for the development of practical hyperthermia methods, which should take into account specific effects accompanying laser light propagation in living tissues including light scattering, blood flow, etc. It is implied that such methods can enable the hyperthermia therapy at a given depth in the tumor area in the absence of traumatic effects on the surrounding healthy tissues. We believe that long-pulse QCW regime looks especially promising taking into account its efficiency and the availability of compact and inexpensive laser diode modules emitting radiation in the tissue transparency window.

\section{Acknowledgements}


This work was partially supported by the Ministry of Science and Higher Education of the Russian Federation (project 16.2969.2017/4.6). The authors also acknowledge contribution of the Russian Science Foundation (Project 19-72-30012) for fabrication of Si-NPs samples and the support from MEPhI Academic Excellence Project (Agreement with the Ministry of Education and Science of the Russian Federation of 27.08.2013, project \#02.a03.21.0005) for providing the research equipment and the related scientific infrastructure. 


\section{References}

[1] L. Canham, Handbook of Porous Silicon, Springer Int. Publ., Switzerland 2014. https://doi.org/10.1007/978-3-319-05744.

[2] A.A. Ischenko, G. V. Fetisov, L.A. Aslanov, Nanosilicon: Properties, synthesis, applications, methods of analysis and control, CRC Press, Boca Raton 2014. https://doi.org/10.1201/b17170.

[3] H.A. Santos, Porous silicon for biomedical applications, Woodhead Publ., 2014. http://www.woodheadpublishing.com/en/book.aspx?bookID=2769.

[4] F. Erogbogbo, K. T. Yong, I. Roy, G. X. Xu, P. N. Prasad, M. T. Swihart, Biocompatible luminescent silicon quantum dots for imaging of cancer cells, ACS Nano 2 (2008) 873-878. https://doi.org/10.1021/nn700319z.

[5] M.B. Gongalsky, L.A. Osminkina, A. Pereira, A.A. Manankov, A.A. Fedorenko, A.N. Vasiliev, V. V. Solovyev, A.A. Kudryavtsev, M. Sentis, A. V. Kabashin, V.Yu. Timoshenko, Laser-synthesized oxide-passivated bright Si quantum dots for bioimaging, Sci. Rep. 6 (2016) 24732. https://doi.org/10.1038/srep24732.

[6] L. Gu, D.J. Hall, Z. Qin, E. Anglin, J. Joo, D.J. Mooney, S.B. Howell, M.J. Sailor, In vivo time-gated fluorescence imaging with biodegradable luminescent porous silicon nanoparticles, Nat. Commun. 4 (2013) 2326. https://doi.org/10.1038/ncomms3326.

[7] V. Yu. Timoshenko, A. A. Kudryavtsev, L. A. Osminkina, A. S. Vorontsov, Yu. V. Ryabchikov, I. A. Belogorokhov, D. Kovalev, P.K. Kashkarov, Silicon nanocrystals as photosensitizers of active oxygen for biomedical applications, JETP Lett. 83 (2006) 423-426. https://link.springer.com/article/10.1134/S0021364006090128.

[8] A.P. Sviridov, V.G. Andreev, E.M. Ivanova, L.A. Osminkina, K.P. Tamarov, V.Yu. Timoshenko, Porous silicon nanoparticles as sensitizers for ultrasonic hyperthermia, Appl. Phys. Lett. 103 (2013) 193110. https://doi.org/10.1063/1.4829148.

[9] K.P. Tamarov, L.A. Osminkina, S. V. Zinovyev, K.A. Maximova, J. V. Kargina, M.B. Gongalsky, Yu. Ryabchikov, A. Al-Kattan, A.P. Sviridov, M. Sentis, A. V. Ivanov, V.N. Nikiforov, A. V. Kabashin, V.Yu. Timoshenko, Radio frequency radiation-induced hyperthermia using Si nanoparticle-based sensitizers for mild cancer therapy, Sci. Rep. 4 (2014) 7034. https://doi.org/10.1038/srep07034.

[10] C. Lee, H. Kim, C. Hong, M. Kim, S.S. Hong, D.H. Lee, W.I. Lee, Porous silicon as an agent for cancer thermotherapy based on near-infrared light irradiation, J. Mater. Chem. 18 (2008) 4790-4795. https://doi.org/10.1039/b808500e. 
[11] D.S. English, L.E. Pell, Z. Yu, P.F. Barbara, B.A. Korgel, Size tunable visible luminescence from individual organic monolayer stabilized silicon nanocrystal quantum dots, Nano Lett. 2 (2002) 681-685. https://doi.org/10.1021/n1025538c.

[12] D. Neiner, H.W. Chiu, S.M. Kauzlarich, Low-temperature solution route to macroscopic amounts of hydrogen terminated silicon nanoparticles, J. Am. Chem. Soc. 128 (2006) 11016-11017. https://doi.org/10.1021/ja064177q.

[13] A. Gupta, M.T. Swihart, H. Wiggers, Luminescent colloidal dispersion of silicon quantum dots from microwave plasma synthesis: Exploring the photoluminescence behavior across the visible spectrum, Adv. Funct. Mater. 19 (2009) 696-703. https://doi.org/10.1002/adfm.200801548.

[14] L. Mangolini, E. Thimsen, U. Kortshagen, High-yield plasma synthesis of luminescent silicon nanocrystals, Nano Lett. 5 (2005) 655-659. https://doi.org/10.1021/n1050066y.

[15] L.A. Osminkina, V.Yu. Timoshenko, Porous Silicon as a Sensitizer for Biomedical Applications, Open Mater. Sci. 3 (2016) 39-48. https://doi.org/10.1515/mesbi-2016-0005.

[16] S. Koynov, R.N. Pereira, I. Crnolatac, D. Kovalev, A. Huygens, V. Chirvony, M. Stutzmann, P. De Witte, Purification of nano-porous silicon for biomedical applications, Adv. Eng. Mater. 13 (2011) B225-B233. https://doi.org/10.1002/adem.201080091.

[17] A. V. Kabashin, M. Meunier, R. Leonelli, Photoluminescence characterization of Sibased nanostructured films produced by pulsed laser ablation, J. Vac. Sci. Technol. B Microelectron. Nanom. Struct. 19 (2001) 2217-2222. https://doi.org/10.1116/1.1420494.

[18] A. V. Kabashin, M. Meunier, Laser-induced treatment of silicon in air and formation of $\mathrm{Si} / \mathrm{SiO}$ x photoluminescent nanostructured layers, in: Mater. Sci. Eng. B Solid-State Mater. Adv. Technol. 101 (2003) 60-64. https://doi.org/10.1016/S0921-5107(02)00651-7.

[19] D. Rioux, M. Laferrière, A. Douplik, D. Shah, L. Lilge, A. V. Kabashin, M.M. Meunier, Silicon nanoparticles produced by femtosecond laser ablation in water as novel contaminationfree photosensitizers, J. Biomed. Opt. 14 (2009) 021010. https://doi.org/10.1117/1.3086608.

[20] P. Blandin, K.A. Maximova, M.B. Gongalsky, J.F. Sanchez-Royo, V.S. Chirvony, M. Sentis, V.Yu. Timoshenko, A. V. Kabashin, Femtosecond laser fragmentation from waterdispersed microcolloids: Toward fast controllable growth of ultrapure Si-based nanomaterials for biological applications, J. Mater. Chem. B 1 (2013) 2489-2495.

https://doi.org/10.1039/c3tb20285b.

[21] R. Intartaglia, A. Barchanski, K. Bagga, A. Genovese, G. Das, P. Wagener, E. Di Fabrizio, A. Diaspro, F. Brandi, S. Barcikowski, Bioconjugated silicon quantum dots from onestep green synthesis. Nanoscale 4 (2012) 1271-1274. https://doi.org/10.1039/C2NR11763K. 
[22] A. Al-Kattan, Y. V. Ryabchikov, T. Baati, V. Chirvony, J.F. Sánchez-Royo, M. Sentis, D. Braguer, V.Yu. Timoshenko, M.A. Estève, A. V. Kabashin, Ultrapure laser-synthesized Si nanoparticles with variable oxidation states for biomedical applications, J. Mater. Chem. B. 4 (2016) 7852-7858. https://doi.org/10.1039/c6tb02623k.

[23] T. Baati, A. Al-Kattan, M.A. Esteve, L. Njim, Yu. Ryabchikov, F. Chaspoul, M. Hammami, M. Sentis, A. V. Kabashin, D. Braguer, Ultrapure laser-synthesized Si-based nanomaterials for biomedical applications: In vivo assessment of safety and biodistribution, Sci. Rep. 6 (2016) 25400. https://doi.org/10.1038/srep25400.

[24] A. V. Kabashin, V.Yu. Timoshenko, What theranostic applications could ultrapure laser-synthesized Si nanoparticles have in cancer? Nanomedicine. 11 (2016) 2247. https://doi.org/10.2217/nnm-2016-0228.

[25] A. V. Kabashin, A. Singh, M.T. Swihart, I.N. Zavestovskaya, P.N. Prasad, Laser Processed Nanosilicon: A Multifunctional Nanomaterial for Energy and Healthcare, ACS Nano. 13 (2019) 9461-9867. https://doi.org/10.1021/acsnano.9b04610.

[26] A.Yu. Kharin, V. V. Lysenko, A. Rogov, Y. V. Ryabchikov, A. Geloen, I. Tishchenko, O. Marty, P.G. Sennikov, R.A. Kornev, I.N. Zavestovskaya, A. V. Kabashin, V.Y. Timoshenko, Bi-Modal Nonlinear Optical Contrast from Si Nanoparticles for Cancer Theranostics, Adv. Opt. Mater. 7 (2019) 1801728. https://doi.org/10.1002/adom.201801728.

[27] V.M. Petriev, V.K. Tischenko, A.A. Mikhailovskaya, A.A. Popov, G. Tselikov, I. Zelepukin, S.M. Deyev, A.D. Kaprin, S. Ivanov, V.Yu. Timoshenko, P.N. Prasad, I.N. Zavestovskaya, A. V. Kabashin, Nuclear nanomedicine using Si nanoparticles as safe and effective carriers of Re-188 radionuclide for cancer therapy, Sci. Rep. 9 (2019) 2017. https://doi.org/10.1038/s41598-018-38474-7.

[28] C. Hong, J. Lee, H. Zheng, S.-S. Hong, C. Lee, Porous silicon nanoparticles for cancer photothermotherapy, Nanoscale Res. Lett. 6 (2011) 321-328. https://doi.org/10.1186/1556$\underline{276 X-6-321}$

[29] V. V. Bezotosnyi, V.Y. Bondarev, O.N. Krokhin, G.T. Mikaelyan, V.A. Oleshchenko, V.F. Pevtsov, Y.M. Popov, E.A. Cheshev, Laser diodes emitting up to $25 \mathrm{~W}$ at $808 \mathrm{~nm}$, Quantum Electron. 39(3) (2009) 241. https://doi.org/10.1070/qe2009v039n03abeh013979.

[30] H. Plattner, My favorite cell - Paramecium, BioEssays 24 (2002) 649-658. https://doi.org/10.1002/bies.10112.

[31] M. Fujishima, M. Kawai, R. Yamamoto, Paramecium caudatum acquires heat-shock resistance in ciliary movement by infection with the endonuclear symbiotic bacterium 
Holospora obtusa, FEMS Microbiol. Lett. 1 (2005) 101-105. https://doi.org/10.1016/i.femsle.2004.11.053.

[32] S. Krenek, T.U. Berendonk, T. Petzoldt, Thermal performance curves of Paramecium caudatum: A model selection approach, Eur. J. Protistol. 2 (2011) 124-137. https://doi.org/10.1016/j.ejop.2010.12.001.

[33] D.M. Pace, K.K. Kimura, The effect of temperature on respiration in Paramecium aurelia and Paramecium caudatum, J. Cell. Comp. Physiol. 24 (1944) 173-183. https://doi.org/10.1002/jep.1030240303.

[34] G. Baronzio, G. Parmar, M. Ballerini, A. Szasz, A brief overview of hyperthermia in cancer treatment, J. Integr. Oncol. 3 (2014) 1-10. https://doi.org/10.4172/2329-6771.1000115.

[35] J.-H. Park, L. Gu, G. von Maltzahn, E. Ruoslahti, S. N. Bhatia, M. J. Sailor, Biodegradable luminescent porous silicon nanoparticles for in vivo applications. Nat. Mater. 8 (2009) 331-336. https://doi.org/10.1038/nmat2398 


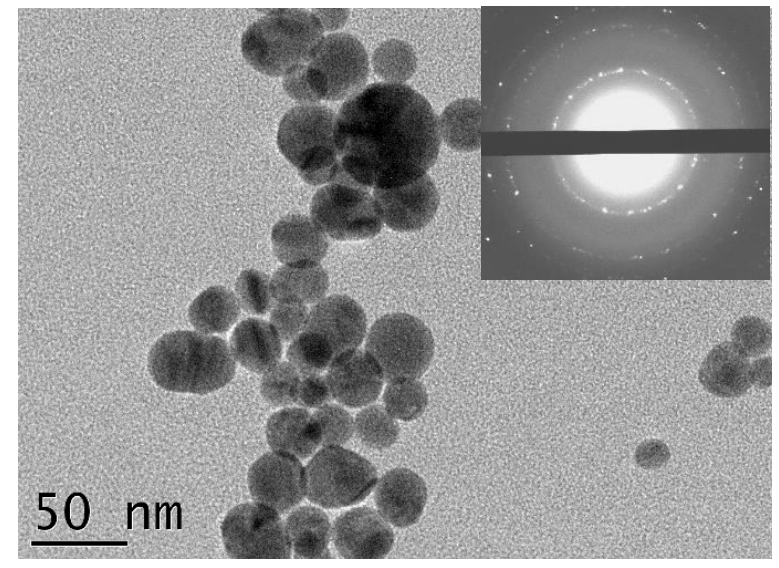

(a)

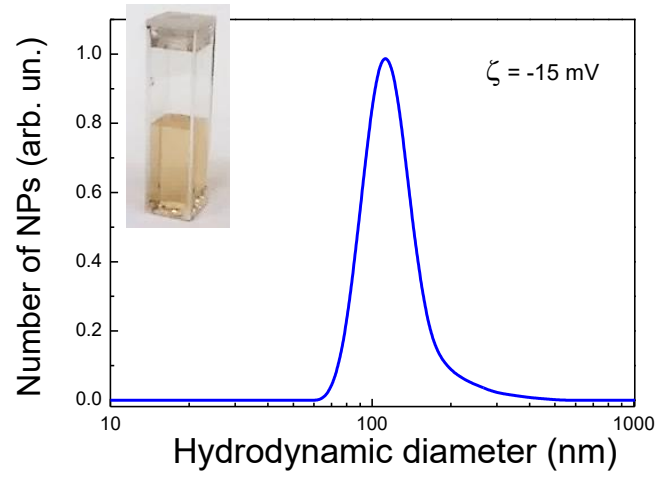

(b)

Figure 1. (a) A TEM image of laser-synthesized Si-NPs. An inset shows a corresponding electron diffraction pattern; (b) Size distribution of Si NPs in aqueous suspension measured by DLS. An inset shows the digital image of a plastic cuvette with aqueous suspension of SiNPs.

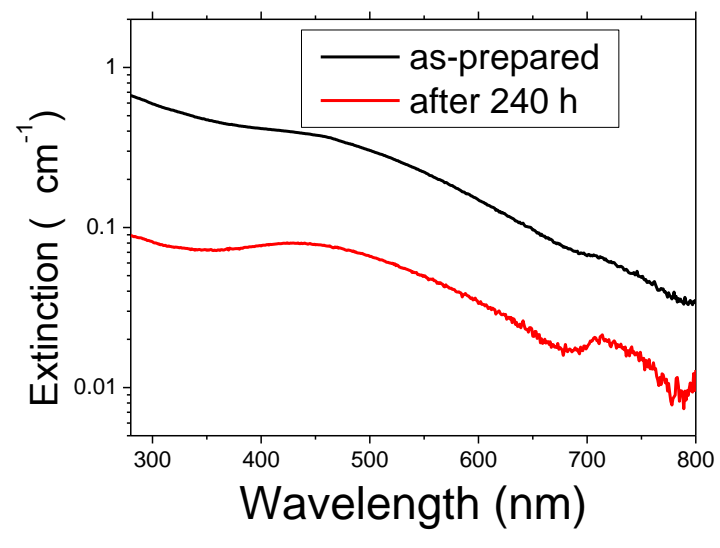

(a)

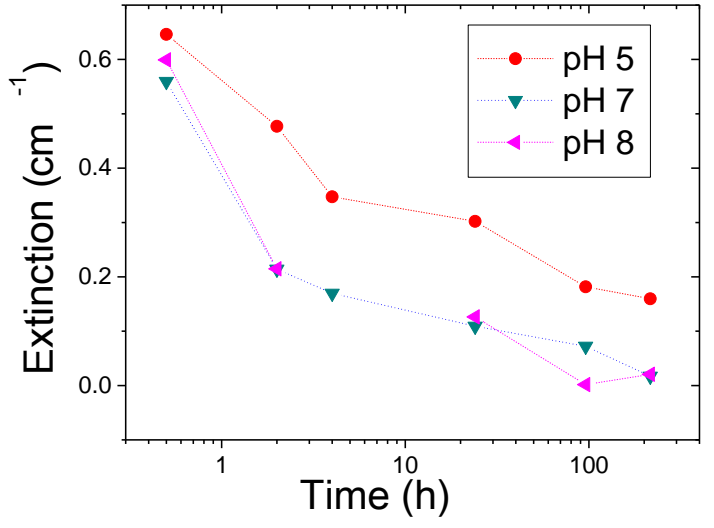

(b)

Figure 2. (a) Extinction spectra of as-prepared aqueous suspension of Si-NPs with concentration of $0.1 \mathrm{~g} / \mathrm{L}$ (black curve) and from the same sample after 10 days of storage in aqueous pH7 buffer (red). (b) Dependences of the Si-NPs extinction at $300 \mathrm{~nm}$ versus time 
of the storage in aqueous media with different $\mathrm{pH}$-levels, those values are marked near the corresponding curves.
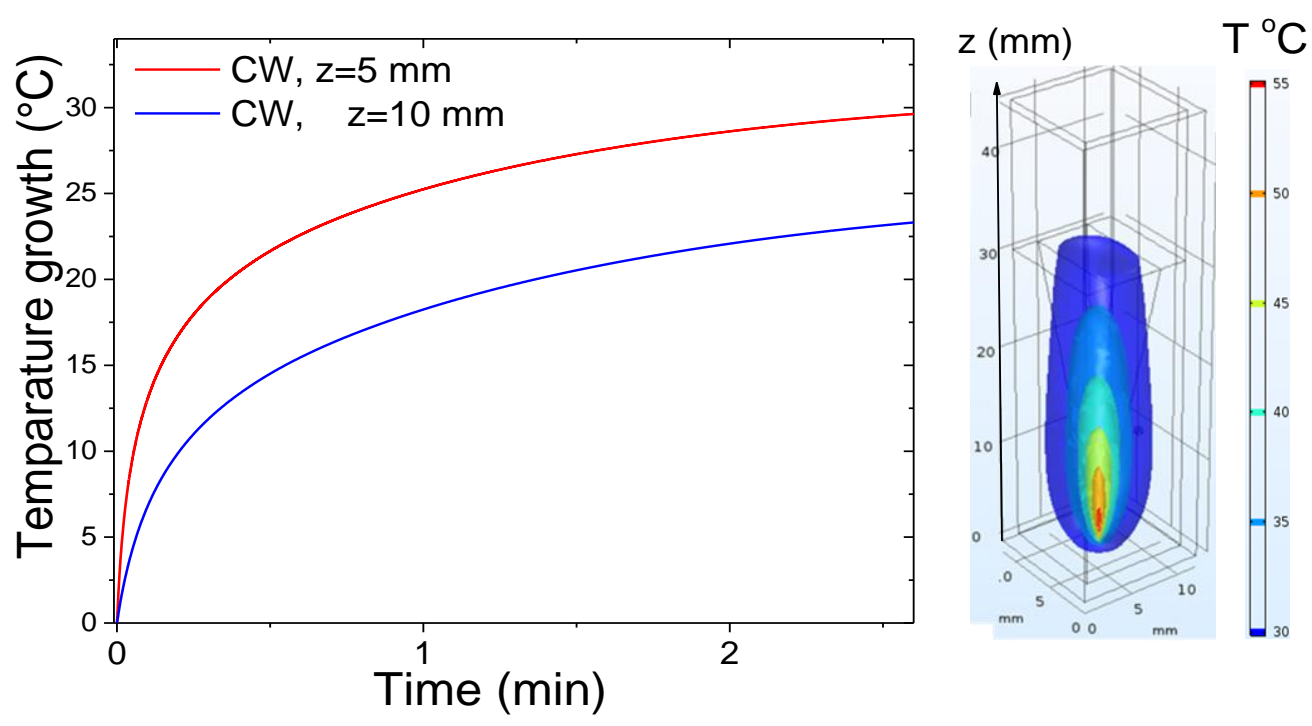

(a)
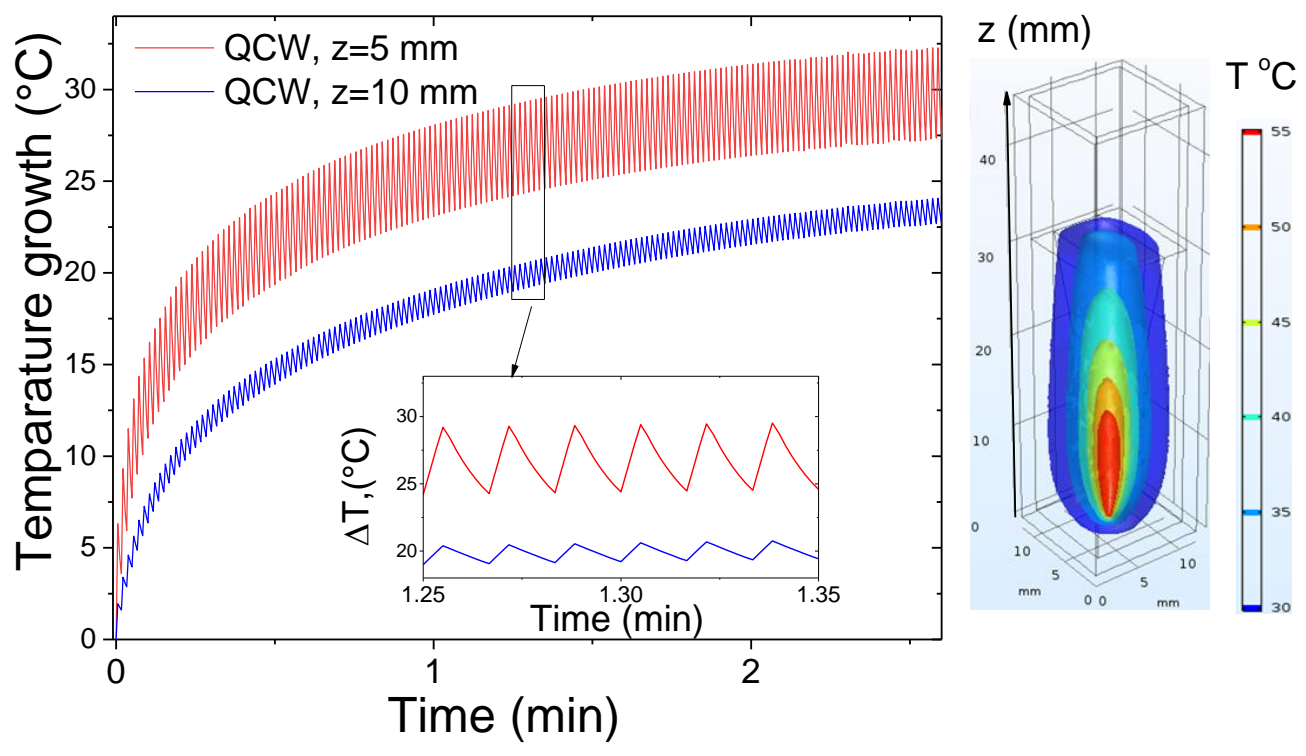

(b)

Figure 3. Calculated temperature growth at two points having the coordinate of $z=5 \mathrm{~mm}$ (red color) and $10 \mathrm{~mm}$ (blue color) from the bottom of a cuvette filled with an aqueous suspension of Si-NPs under irradiation by a laser diode at $808 \mathrm{~nm}$ in CW (a) and QCW (b) regimes. Inserts in panels (a) and (b) show 3D-isothermal surfaces in the cuvette after 
irradiation for 5 min in CW and QCW modes, respectively. An additional inset in panel (b) shows an enlarged part of temperature transients after the first minute of the irradiation.

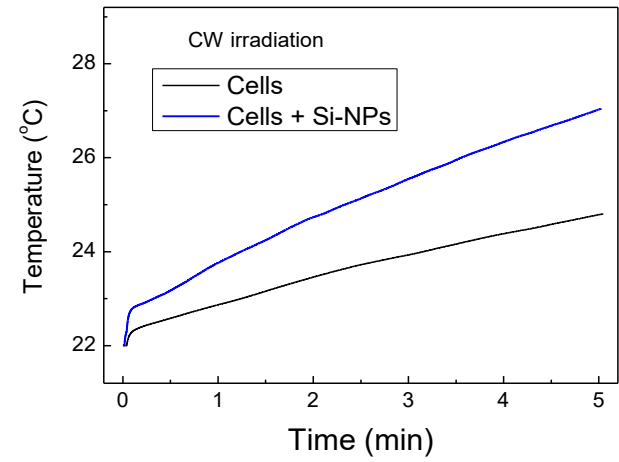

(a)

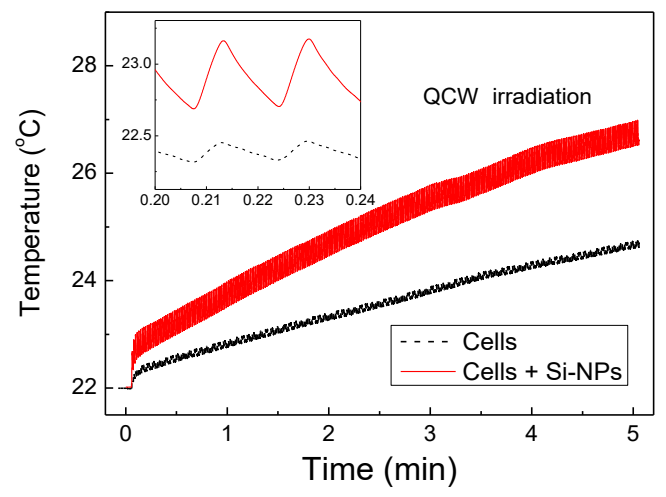

(b)

Figure 4. (a) Temperature transients for aqueous suspensions of Paramecium Caudatum cells (black curve) and those with incubated Si-NPs (blue curve) under CW laser irradiation. (b) Temperature transients for the same cell suspensions (black curve) and those with incubated Si-NPs (red curve) under QCW laser irradiation (pulse duration $\tau=0.3 \mathrm{~s}$, period $\mathrm{T}=1 \mathrm{~s})$. An inset in panel (b) shows parts of these transients expanded into the time interval of $0.2-0.24 \mathrm{~s}$.

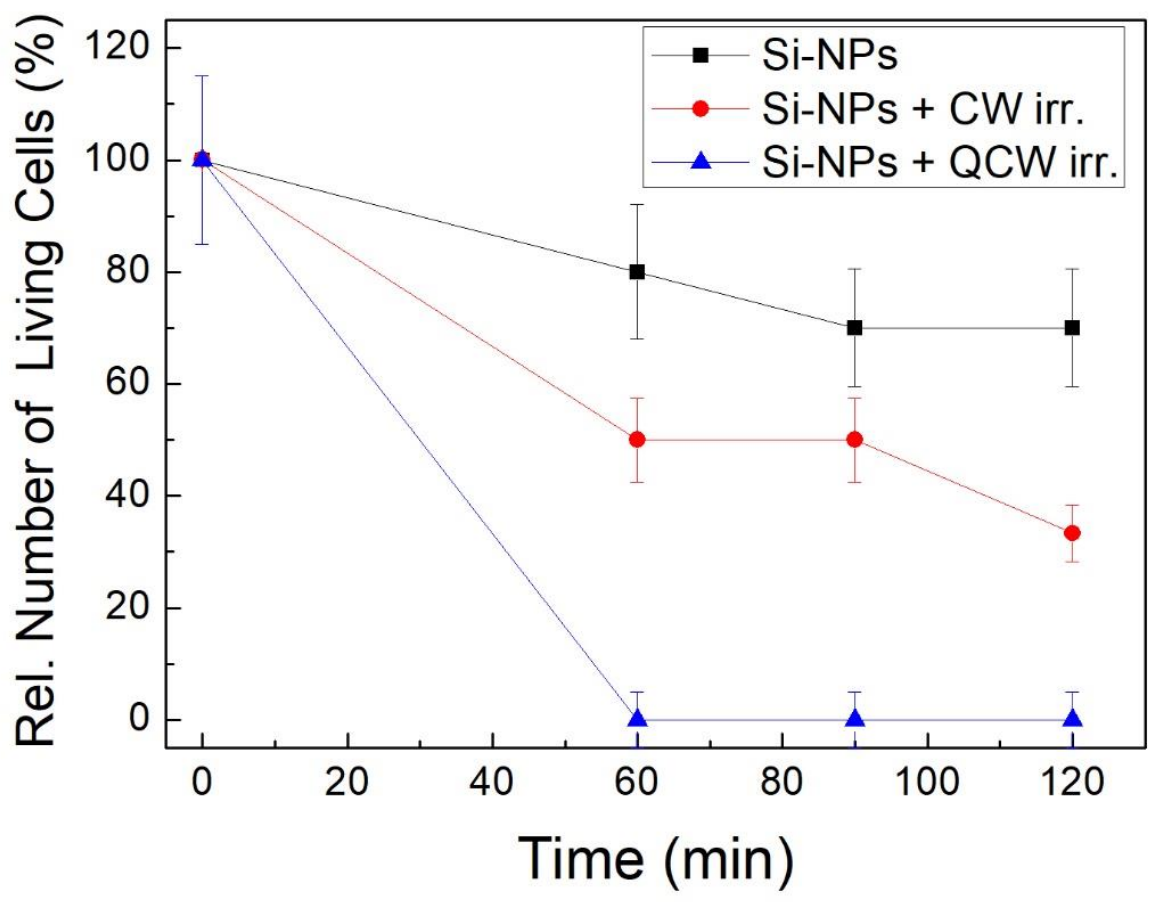


Figure 5. Relative number of living cells of Paramecium Caudatum versus time after injection of Si-NPs (black squares), followed with the irradiation in CW (red circles) and QCW (blue triangles) regimes (808 $\mathrm{nm}$, mean power $3 \mathrm{~W}$ ).

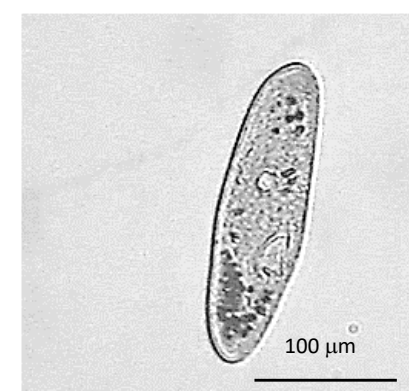

(a)

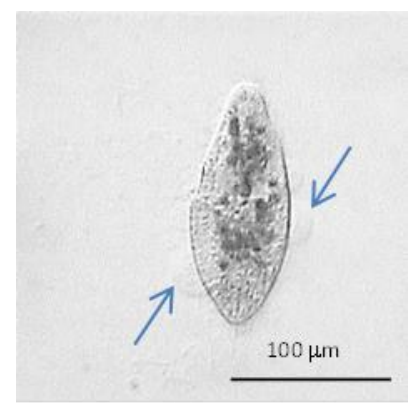

(b)

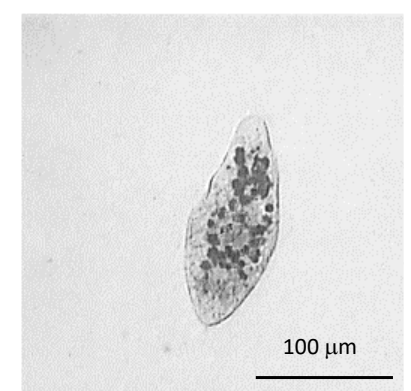

(c)

Figure.6. Digital images of unicellular organisms Paramecium Caudatum in aqueous suspension of Si-NPs (a) before exposition to laser beam, (b) $1 \mathrm{~min}$ after laser irradiation, (c) 3 min after laser irradiation. Arrows in panel (b) indicate bubbles near the perforated membrane. The laser irradiation was done at $808 \mathrm{~nm}$ in QCW regime for $15 \mathrm{~s}$. The initial temperature was $22.5^{\circ} \mathrm{C}$. 